\title{
Considerations for Stress Testing Performed in Conjunction with Myocardial Perfusion Imaging
}

\author{
April Mann, MBA, CNMT, NCT, RT(N), FSNMMI-TS ${ }^{1}$, and Jessica Williams, CNMT, RT(N), FSNMMI-TS ${ }^{2}$ \\ ${ }^{1}$ Martin Imaging, Inc., Destin, Florida; and ${ }^{2} H C A$ Healthcare, London, England
}

CE credit: For CE credit, you can access the test for this article, as well as additional JNMT CE tests, online at https://www.snmmilearningcenter.org. Complete the test online no later than June 2023. Your online test will be scored immediately. You may make 3 attempts to pass the test and must answer $80 \%$ of the questions correctly to receive $1.0 \mathrm{CEH}$ (Continuing Education Hour) credit. SNMMI members will have their CEH credit added to their VOICE transcript automatically; nonmembers will be able to print out a CE certificate upon successfully completing the test. The online test is free to SNMMI members; nonmembers must pay $\$ 15.00$ by credit card when logging onto the website to take the test.

For myocardial perfusion imaging (MPI), the best test to evaluate hemodynamic changes during stress is an exercise treadmill test. It provides independent prognostic value, including evaluation of total exercise time, performance, and capacity; heart rate response during exercise, with ischemia, and in recovery; blood pressure response; myocardial oxygen demand; and assessment of symptoms. Combining these exercise data with perfusion imaging provides the best prognostic value and risk stratification for patients. Although exercise stress testing accompanied by MPI is preferential, it is not always possible since an increasing number of patients cannot exercise to a maximal (symptom-limited) level. Further, there is much evidence in the literature demonstrating a suboptimal, non-symptom-limited (not achieving at least $4-6 \mathrm{~min}$ or $<85 \%$ of maximum predicted heart rate) exercise test performed as part of an MPI study may result in a false-negative outcome. Therefore, pharmacologic stress agents provide an excellent alternative for those patients who cannot achieve an adequate heart rate response or adequately perform physical exercise. This article focuses on considerations for performing stress (exercise and pharmacologic) testing in conjunction with MPI. It is meant to provide a basic overview of the principles of exercise stress testing; discuss the indications, contraindications, patient preparation, and protocols for exercise stress testing; discuss the contraindications, administration protocols, and side effects for vasodilator (adenosine, dipyridamole, and regadenoson) stress testing; and discuss the contraindications, administration protocols, and side effects for dobutamine stress testing.

Key Words: stress testing; exercise; dipyridamole; adenosine; regadenoson; dobutamine

J Nucl Med Technol 2020; 48:114-121

DOI: 10.2967/jnmt.120.245308

Received Mar. 19, 2020; revision accepted Apr. 3, 2020.

For correspondence or reprints contact: April Mann, Martin Imaging, Inc., 47 Hadley Village Rd., South Hadley, MA 01075.

E-mail: april@martinimaging.com

Published online Apr. 10, 2020.

COPYRIGHT (C) 2020 by the Society of Nuclear Medicine and Molecular Imaging.
$\mathbf{F}$ or myocardial perfusion imaging (MPI), the best way to evaluate hemodynamic changes during stress is through an exercise treadmill test (1-8). It provides independent prognostic value, including evaluation of total exercise time, performance, and capacity; heart rate response during exercise, with ischemia, and in recovery; blood pressure response; myocardial oxygen demand; and symptoms. Combining these exercise data with perfusion imaging results provides the best prognostic value and risk stratification for patients.

\section{PRINCIPLES OF EXERCISE TESTING}

Stress testing is a commonly used method to determine and assess the performance of the body and heart $(1,2,6)$. When measuring engine performance, one may consider horsepower (the amount of work done) and fuel consumption (liters of gas consumed) as endpoints. Similarly, in humans, performance can be measured as fuel consumption in terms of the amount of oxygen consumed $(1,6)$. The 2 endpoints used to measure oxygen consumption are external work rate (total body consumption $\left[\mathrm{VO}_{2}\right]$ ) and internal work rate (myocardial consumption $\left.\left[\mathrm{MVO}_{2}\right]\right)$.

$\mathrm{VO}_{2}$ is a measure of volume $(\mathrm{V})$ of oxygen $\left(\mathrm{O}_{2}\right)$ consumed by the body, also known as maximal oxygen uptake and reported as $\mathrm{VO}_{2} \max (1,6)$. It is defined as measurement of the maximum amount of oxygen a person can consume (metabolize) during physical exercise. $\mathrm{VO}_{2}$ is measured by calculating cardiac output $\times$ arteriovenous oxygen differential $\left(\mathrm{A}-\mathrm{V} \mathrm{O}_{2}\right.$ ) (Table 1). Normal $\mathrm{VO}_{2}$ values are $35-40 \mathrm{~mL} / \mathrm{kg} / \mathrm{min}$ for sedentary men and $27-30 \mathrm{~mL} / \mathrm{kg} / \mathrm{min}$ for sedentary women. It is commonly used to measure the aerobic endurance of athletes during their course of intensive training.

Cardiac output is defined as the volume of blood the heart pumps per minute $(1,6)$. It is calculated by multiplying stroke volume by heart rate (cardiac output $=$ heart rate $\times$ stroke volume) (Table 1). Normal cardiac output in healthy patients ranges from 4.0 to $8.0 \mathrm{~mL} / \mathrm{min}$. Stroke volume is the amount of blood pumped from the left ventricle per beat and is determined by the preload, contractility, and afterload of the 
TABLE 1

Cardiac Measurement Terms, Equations, and Reference Values

\begin{tabular}{|c|c|c|}
\hline Term & Formula & Range \\
\hline $\mathrm{VO}_{2} /$ maximal $\mathrm{VO}_{2}$ & Cardiac output $\times \mathrm{A}-\mathrm{V} \mathrm{O}_{2}$ differential & $\begin{array}{l}35-40 \mathrm{~mL} / \mathrm{kg} / \mathrm{min} \text { (men); } \\
27-30 \mathrm{~mL} / \mathrm{kg} / \mathrm{min} \text { (women) }\end{array}$ \\
\hline Cardiac output & Heart rate $\times$ stroke volume & $4.0-8.0 \mathrm{~mL} / \mathrm{min}$ \\
\hline Stroke volume & End-diastolic volume - end-systolic volume & $60-100 \mathrm{~mL}$ \\
\hline A- $\mathrm{V} \mathrm{O}_{2}$ differential & $\begin{array}{l}\text { Oxygen content of pulmonary blood - oxygen } \\
\text { content in mixed venous blood }\end{array}$ & $4.5 \mathrm{~mL} / 100 \mathrm{~mL} / \mathrm{min}$ \\
\hline $\mathrm{MVO}_{2} /$ rate-pressure product & $\begin{array}{l}\text { Coronary blood flow } \times \mathrm{A}-\mathrm{V} \mathrm{O}_{2} \\
\text { differential/heart rate } \times \text { systolic blood pressure }\end{array}$ & $30-35 \mathrm{~mL} / \mathrm{min}$ \\
\hline MPHR & 220 - age & Not applicable \\
\hline
\end{tabular}

myocardium. Stroke volume is calculated by subtracting end-systolic volume (ESV) from end-diastolic volume (EDV) (Table 1). In healthy patients, standard normal values are $60-100 \mathrm{~mL}$. A higher exercise tolerance results in a higher stroke volume, providing a better $\mathrm{VO}_{2}$ measurement. $\mathrm{A}-\mathrm{V} \mathrm{O}_{2}$ differential is the measurement of the arteriovenous oxygen difference (Table 1) $(1,6)$. It is defined as the difference in the oxygen content between the arterial blood and the venous blood, and it is used to measure the amount of oxygen removed from the blood in capillaries as the blood circulates through the body. The normal value for the $\mathrm{A}-\mathrm{V} \mathrm{O}_{2}$ differential is $4.5 \mathrm{~mL} / 100 \mathrm{~mL} / \mathrm{min}$.

$\mathrm{MVO}_{2}$ is equal to coronary blood flow multiplied by the A- $-\mathrm{O}_{2}$ differential $(1,6)$. During diastole, the ventricles receive blood before the systolic contraction occurs. This filling phase of the cardiac cycle allows the coronary arteries to provide maximum blood flow to the heart. $\mathrm{MVO}_{2}$ consumption may also be calculated using heart rate $\times$ systolic blood pressure, which is referred to as the rate-pressure product (Table 1) $(1,6)$. Normal $\mathrm{MVO}_{2}$ is $30-35 \mathrm{~mL} /$ min for an average-sized $(\sim 300 \mathrm{~g})$ healthy heart.

During exercise testing, systolic blood pressure is expected to go up along with oxygen consumption. Systolic pressure equals flow $\times$ resistance of the vessels as cardiac output is increasing and peripheral resistance is decreasing. In healthy individuals, systolic blood pressure should increase during exercise to maintain an adequate cardiac output. A fall in blood pressure during exercise means a leveling or decrease in cardiac output. This decrease may indicate coronary artery disease (CAD). Therefore, an increase in systolic blood pressure is normal during exercise and a decrease may indicate a poor prognosis and outcomes $(1,2,6)$.

Heart rate response to stress is also an important measure during exercise testing. Heart rate should increase during exercise, and patients should be able to achieve at least $85 \%$ of their maximum predicted heart rate (MPHR) $(1,2,6-8)$. The MPHR should be calculated by subtracting the patient age from 220 (Table 1) (1,2,6-8). However, since the MPHR measure at the $95 \%$ confidence limit is 45 beats per minute (MPHR for a 60-y-old patient ranges from 137 to 182 beats per minute, making it difficult to accurately deter- mine $85 \%$ MPHR target heart rate), it is important to always perform a symptom-limited exercise test rather than use achievement of $85 \%$ MPHR as the sole reason to terminate testing.

It is important to monitor heart rate recovery after exercise testing. Heart rates that are slow to return to baseline during recovery may indicate chronotropic incompetence. Chronotropic incompetence is the inability of the heart to adequately respond to increased demands during exercise and also results in the heart's inability to recover from this demand after exercise $(1,6)$. It most often occurs in patients with $\mathrm{CAD}$ and congestive heart failure, resulting in exercise intolerance, and may impair quality of life. It is considered an independent predictor of risk for major adverse complications and cardiac events (death and myocardial infarction) in patients with CAD. In addition to chronotropic incompetence, low heart rates during stress may indicate large cardiac dimensions commonly seen in athletes who perform routine intense training. This condition does not present a concern for future cardiac events and is considered normal in those patients $(1,2,6)$.

Metabolic equivalent (MET) is another measure achieved and recorded during exercise testing. One MET equals approximately $3.5 \mathrm{~mL}$ of oxygen $/ \mathrm{kg}$ of body weight $/ \mathrm{min}^{2}$ $(1,6)$. It is well established most healthy, sedentary people seldom exercise beyond 10-11 METs $(1,2,6)$. Further, in most patients with CAD, workloads of 8 METs are sufficient for angina evaluation. This measure is also used to determine the functional classes of patients and assist in determining treatment options $(1,2,6-8)$. Class I is defined as patients able to exercise beyond 7 or 8 METs, class II patients become symptom-limited at 5 or 6 METs, and class III patients usually becomes symptom-limited at 3-4 METs.

Exercise testing is a valid cardiac measure of performance and capacity. The heart extracts approximately $70 \%$ of the oxygen in the blood at rest, and increasing the extraction rate cannot significantly increase the delivery of oxygen. Therefore, coronary blood flow must increase in order to increase the myocardial oxygen supply $(1,2,6-8)$. In healthy people, coronary blood flow increases proportionally to the increased demand for oxygen by the 
myocardium. Myocardial ischemia is caused when coronary blood flow cannot meet the demand for oxygen, resulting in symptoms such as angina (chest pain) and shortness of breath. During an exercise test, ischemia may manifest as anginal pain, ST-segment or T-wave changes, ventricular dysfunction, arrhythmias, or any combination of these findings $(1,2,6)$.

\section{PERFORMING AN EXERCISE STRESS TEST}

\section{Preparations, Indications, and Contraindications}

Standard stress protocols including patient preparation should be performed in compliance with American College of Cardiology/American Heart Association/American Society of Nuclear Cardiology guidelines for stress testing. Preparation includes having the patient fast (nil per os) for $3 \mathrm{~h}$ and withhold caffeine for $12 \mathrm{~h}$ before testing $(2,3,6-8)$. In addition, a larger-bore ( $>24$ gauge) intravenous cannula should be inserted, and leads for 12lead electrocardiogram monitoring should be placed. Some cardiac medications have been demonstrated to diminish the diagnostic accuracy of exercise testing. However, physicians may sometimes order the procedure to evaluate the efficacy of these medications $(3,6)$. Therefore, discontinuation of cardiac medications should be at the discretion of the referring physician.

When performing stress tests in conjunction with MPI, it is necessary to have at least 2 qualified individuals present during the procedure, one to monitor the patient and the other to inject the radiotracer $(3,6-8)$. There are several reasons to perform an exercise stress test. However, the standard clinical indications for testing include evaluation of patients with chest pain or other findings suggestive of CAD, determination of prognosis and severity of disease, evaluation of effects from medical and surgical therapy, screening for latent CAD (only $\sim 30 \%$ of patients with ischemia have chest pain), and evaluation of arrhythmias, functional capacity, or congenital heart disease $(1-3,6-8)$. More specifically, some of the common clinical indications for stress MPI include diagnosis of suspected CAD, risk stratification of known CAD, assessment of medical or surgical therapies used to treat known CAD, preoperative assessment in patients with cardiac symptoms, and myocardial viability assessment $(2,3,6-8)$.

Before performing an exercise test, it is necessary to ensure the patient has no known contraindications-neither relative nor absolute. A relative contraindication is a clinical situation in which the test may be performed, but with caution. Examples include clinically significant noncardiac disorders, significant physical handicaps, debilitation or old age, mental instability or noncooperation, severe anemia or high fever, moderate to severe hypertension, pulmonary hypertension, moderate aortic stenosis, known significant left main coronary artery disease, asymptomatic severe aortic stenosis, other serious heart diseases, tachyarrhythmias, bradyarrhythmias, and, if performed in conjunction with imaging, left bundle branch block (LBBB), permanent pacemaker, or ventricular preexcitation $(1-3,6-8)$.

Absolute contraindications are clinical situations in which an exercise test should not be performed. These include acute myocardial infarction (within 2-4 d), unstable or crescendo angina, serious cardiac arrhythmias, acute myocarditis or pericarditis, severe aortic stenosis, acute or severe congestive heart failure, cardiogenic shock, acute pulmonary embolism or infarction, aortic dissection, any acute or serious noncardiac disorder, severe hypertension or hypotension, and severe physical handicaps $(1-3,6-8)$.

\section{Protocols}

Various protocols are used to perform physical exercise tests including Bruce, modified Bruce, Naughton, and Chung (1-3,6-8). Some practitioners may also decide to perform a manual test (customized at the time of the test for speed, grade, and time per stage and not automated) if there is a specific clinical scenario they would like to reproduce or evaluate. However, manual testing should be performed in very limited situations and with extreme caution since there are no documented specific endpoints for reference or comparison.

For MPI, the Bruce protocol is routinely chosen in most laboratories (Table 2). It is the recommended protocol for use in conjunction with MPI in various guidelines and is well validated in the published literature $(1,6-8)$. During the procedure and as recommended in the guidelines, it is necessary to measure and document the patient's heart rate and blood pressure at approximately $2 \mathrm{~min}$ into every stage, at peak exercise, and for at least $4 \mathrm{~min}$ into the recovery phase $(1-3,6-8)$. The electrocardiogram should be monitored continuously during the test and for at least $4 \mathrm{~min}$ into the recovery phase and recorded during each stage of exercise, at peak exercise, at termination of the recovery phase, and whenever any clinical abnormalities that may occur. (1-3,6-8). The radiotracer should be injected as close to peak exercise as possible and approximately $1 \mathrm{~min}$ before the patient is no longer able to continue.

The endpoint of exercise should be symptom-limited (moderate to severe chest pain, excessive shortness of breath, fatigue). The achievement of $85 \% \mathrm{MPHR}$ is not

TABLE 2

Bruce Protocol

\begin{tabular}{cccc}
\hline Stage & Minutes & Speed $(\mathrm{mph})$ & Grade $(\%)$ \\
\hline 1 & 3 & 1.7 & 10 \\
2 & 6 & 2.5 & 12 \\
3 & 9 & 3.4 & 14 \\
4 & 12 & 4.2 & 16 \\
5 & 15 & 5.0 & 18 \\
6 & 18 & 5.5 & 20 \\
7 & 21 & 6.0 & 22 \\
\hline
\end{tabular}

$1 \mathrm{mile}=1.6 \mathrm{~km}$ 
considered the sole indication for termination of the test (3). Patients with known CAD, and more specifically if the test is being performed for evaluating symptom management of medical therapies, the prognostic value of the test is preserved without reaching $85 \%$ MPHR (3). If patients are known to have a limited exercise capacity, a modified Bruce protocol may be considered as an alternative (Table 3).

\section{Termination of Testing}

As discussed earlier, during a MPI study, the radiotracer should be injected as close to peak exercise as possible and approximately $1 \mathrm{~min}$ prior to when the patient is no longer able to continue. There are several reasons to consider early termination of the exercise testing. The first and most important reason is the patient indicates the need to stop. Other reasons include atrial tachycardia, atrial fibrillation, or atrial flutter; onset of second- or third-degree heart block; progressive anginal pain; severe ST depression $(>3 \mathrm{~mm})$; ST elevation of more than $2 \mathrm{~mm}$ in the precordial or inferior leads without a resting $\mathrm{Q}$ wave; development of preventricular contractions in pairs or with increasing frequency as exercise increases or when ventricular tachycardia develops (runs of 3 or more preventricular contractions); a progressive drop in heart rate or systolic blood pressure; dyspnea, fatigue, or faintness; severe musculoskeletal pain; extreme elevations in systolic or diastolic blood pressure associated with headache or blurred vision; malfunctioning equipment; an uninterruptable electrocardiogram tracing; and undetectable leads $(1-3,6-8)$.

\section{VASODILATOR AND IONOTROOPIC STRESS TESTING}

Although exercise stress testing accompanied by MPI is preferential, it is not always possible since an increasing number of patients cannot exercise to a maximal level. Further, there is much evidence in the literature demonstrating that a suboptimal, non-symptom-limited (not achieving at least $4-6 \mathrm{~min}$ or $<85 \%$ of MPHR) exercise test performed as part of an MPI study may result in a falsenegative outcome $(1,6-8)$. Therefore, pharmacologic stress agents provide an excellent alternative for those patients who cannot achieve an adequate heart rate response or adequately perform physical exercise. Patient preparation,

TABLE 3

Modified Bruce Protocol

\begin{tabular}{lccc}
\hline Stage & Minutes & Speed $(\mathrm{mph})$ & Grade $(\%)$ \\
\hline 0 & 3 & 1.7 & 0 \\
$1 / 2$ & 6 & 1.7 & 5 \\
1 & 9 & 1.7 & 10 \\
2 & 12 & 2.5 & 12 \\
3 & 15 & 3.4 & 14 \\
4 & 18 & 4.2 & 16 \\
5 & 21 & 5.0 & 18 \\
6 & 24 & 5.5 & 20 \\
7 & 27 & 6.0 & \\
\multicolumn{1}{l}{1 mile $=1.6 \mathrm{~km}}$. & & \\
\hline
\end{tabular}

monitoring, and clinical indications are similar to those for an exercise stress test.

\section{Adenosine}

Adenosine is a commonly used pharmaceutical for stress testing performed in conjunction with MPI. It is a potent coronary arteriolar vasodilator that acts by directly activating adenosine receptors on cell membrane surfaces. Adenosine occurs naturally in the body and induces vasodilation through specific activation of the $\mathrm{A}_{2 \mathrm{~A}}$ receptor. During infusion, adenosine causes blood flow rates $4-6$ times those at rest. Peak vasodilation occurs at 1-2 min of continuous infusion, and adenosine has a 10-s half-life. In areas of the myocardium supplied by stenotic arteries, an attenuated hyperemic response may occur. Depending upon the coronary flow reserve limitations and severity of coronary stenosis, a relative fluctuation in flow may be induced. Similar to dipyridamole, adenosine used in conjunction with MPI is well validated in the literature $(3,6-9)$.

Generally, adenosine does not cause myocardial ischemia, as the myocardial blood flow increases to all coronary artery vascular beds with minimal or no rate increase in ratepressure product or myocardial oxygen demand. In a small percentage of patients with severe CAD, true ischemia may be induced due to the coronary steal phenomenon. Coronary steal is an iatrogenic condition characterized by shunting of well-oxygenated blood from a critical area of low perfusion to an area of higher perfusion. Since tracer uptake in the myocardium is proportional to the regional myocardial blood flow, a heterogeneous distribution of radiotracer occurs in the myocardium when coronary steal occurs (3,6-9).

Adenosine is used in patients with baseline electrocardiogram abnormalities such as a LBBB, ventricular pacing, and Wolff-Parkinson-White syndrome. It is also used in patients less than $1 \mathrm{~d}$ after an acute myocardial infarction who are otherwise clinically stable and in patients who present in the emergency department following acute coronary syndrome (3,6-9). Adenosine is also used not only in patients who cannot exercise because of pulmonary, peripheral vascular, musculoskeletal, and mental conditions but also in patients who are unwilling to exercise. As with exercise stress testing, $\beta$-blockers, nitrates, and calcium antagonists have been reported to decrease the accuracy of diagnosis with the use of vasodilators before stress testing $(3,6-9)$.

Contraindications to adenosine stress tests include second- or third-degree atrioventricular block in the absence of a pacemaker or sick sinus syndrome, consumption of caffeine or aminophylline within $12 \mathrm{~h}$ of administration, a systolic blood pressure of less than $90 \mathrm{~mm} \mathrm{Hg}$, recent use of dipyridamole or dipyridamole-containing medications (e.g., Aggrenox [aspirin/dipyridamole]; Boehringer Ingelheim), known hypersensitivity to adenosine, unstable acute myocardial infarction or acute coronary artery syndrome, and prior cardiac transplantation. Active wheezing and bronchospasm are clinical situations for which adenosine use is considered an absolute contraindication $(3,6-9)$. However, 
there have been reports of patients with adequately controlled asthma receiving adenosine after being pretreated with 2 doses of albuterol or a comparable inhaler (6-8). Sinus bradycardia with a heart rate of less than 40 beats per minute is considered another relative contraindication to adenosine use.

Adenosine is administered intravenously via an infusion pump over a $4-$ to $6-\mathrm{min}$ period at a rate of $140 \mu \mathrm{g} / \mathrm{kg} / \mathrm{min}$. Because of the very short half-life of adenosine $(10 \mathrm{~s})$, the radiotracer is injected during infusion of the adenosine (Fig. 1) (3,6-9). If a 4-min protocol is being used, the radiotracer should be injected at $2 \mathrm{~min}$, and if a 6-min protocol, at 3 min. Adenosine is supplied in 20- and 30-mL single-dose vials of $3 \mathrm{mg} / \mathrm{mL}$. To calculate the volume of adenosine to be drawn, the patient weight must be converted to kilograms (patient weight in pounds $/ 2.2=$ kilograms). This weight in kilograms is necessary to calculate the dose $(\mathrm{mL})$ needed for infusion $[(\mathrm{kg} \times 0.14 \mathrm{mg} / \mathrm{kg}) \times(6 \mathrm{~min} /[3.0 \mathrm{mg} / \mathrm{m}])=\mathrm{ml}]$. If a 4-min protocol is being used, the time in the dose calculation should be changed accordingly $(3,6-9)$.

To obtain additional data and improve image quality, patients who are ambulatory and do not have a LBBB or a paced ventricular rhythm may walk on the treadmill at 2.74 $\mathrm{km} / \mathrm{h}(1.7 \mathrm{mph})$ with $0 \%$ elevation during the adenosine infusion. When performing this protocol, the radiotracer should be administered at 2 min of walking (4-min protocol) or 3 min of walking (6-min protocol). After injection of the radiotracer, patients should continue walking for an additional 2 min to allow for adequate vasodilation and uptake of the tracer $(3,6-9)$.

During administration of adenosine, early termination should occur if any of the following clinical situation are present: severe hypotension, severe chest pain with ST depression of $2 \mathrm{~mm}$ or greater, complete heart block, symptomatic second-degree heart block, signs of poor perfusion, patient request, or wheezing $(3,6-9)$. If the patient has a seizure, the adenosine infusion must be terminated immediately. Aminophylline should not be administered in this situation, as it may increase the risk of seizure associated with adenosine. Methylxanthine is also not recommended in patients who experience seizures in association with adenosine administration (3,6-9).

Many patients $(80 \%)$ who receive adenosine may experience adverse reactions. However, since adenosine has a very short half-life (10 s), reversal methods are rarely

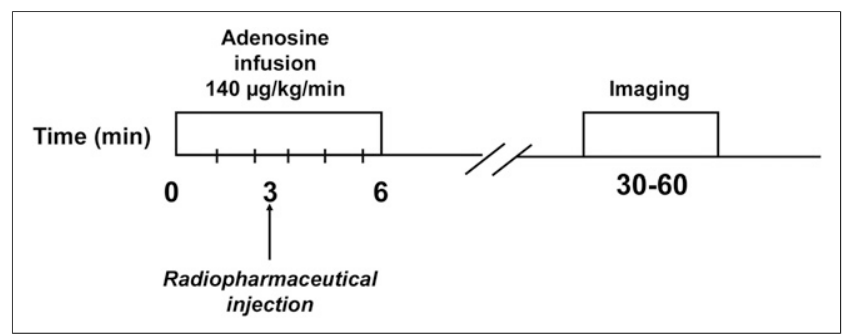

FIGURE 1. Adenosine infusion protocol. needed because the side effects dissipate within a few seconds after the infusion is terminated. Common reactions include flushing, chest discomfort, dyspnea, headache, dizziness, throat or jaw discomfort, and gastrointestinal discomfort (3,6-9). Atrioventricular blocks have been demonstrated to occur in $7.6 \%$ of patients but rarely require termination of the adenosine infusion. ST-segment depression may occur in $5 \%-7 \%$ of the patients, and is indicative of CAD. Fatal and nonfatal myocardial infarction are rare but have also been demonstrated to occur during administration of adenosine. If persistent symptoms or other clinical situations occur, aminophylline may be administered at a dose of 50-250 mg through a slow (50-100 mg over 30$60 \mathrm{~s})$ intravenous injection $(3,6-9)$.

\section{Dipyridamole}

Dipyridamole has the longest history of use of all the vasodilators and has the most data available in the literature referencing its use with MPI. It is a potent coronary vasodilator that acts by blocking intracellular reuptake and deamination of natural adenosine within the body $(3,6-8,10)$. After the infusion of dipyridamole, the level of intrinsic adenosine is increased, causing vascular smooth muscle relaxation and coronary dilation. Blood flow is increased 3-5 times above baseline levels in normal coronary arteries. When coronary stenosis is present, blood flow may be restricted, resulting in a decrease in tracer uptake in the area of the myocardium correlating to the specific coronary artery territory affected. The half-life of dipyridamole is 30-45 $\min (3,6-8,10)$.

Dipyridamole is used in patients with baseline electrocardiogram abnormalities such as LBBB, ventricular pacing, and Wolff-Parkinson-White syndrome. It is also used in patients less than $1 \mathrm{~d}$ following an acute myocardial infarction who are clinically stable; patients who present in the emergency department following acute coronary syndrome; patients who cannot exercise because of pulmonary, peripheral vascular, musculoskeletal, or mental conditions; and patients who are unwilling to exercise. As with exercise stress testing, $\beta$-blockers, nitrates, and calcium antagonists have been reported to decrease the accuracy of diagnosis with the use of vasodilators in stress testing $(3,6-8,10)$. The contraindications to dipyridamole include second- and third-degree atrioventricular block in the absence of a pacemaker or sick sinus syndrome, consumption of caffeine or aminophylline within $12 \mathrm{~h}$ before administration, systolic blood pressure of less than $90 \mathrm{~mm} \mathrm{Hg}$, asthma with wheezing, and sinus bradycardia with a heart rate of less than 40 beats per minute $(3,6-8,10)$.

Dipyridamole is administered via an infusion pump at $0.56 \mathrm{mg} / \mathrm{kg}$ over a $4-\mathrm{min}$ period $(142.0 \mu \mathrm{g} / \mathrm{kg} / \mathrm{min})$. It may also be administered manually through an intravenous line at a rate of $10 \mathrm{~mL} / \mathrm{min}$ when a $40-\mathrm{mL}$ volume is used. The radiotracer is injected at 3-5 min after infusion (Fig. 2) $(3,6-8,10)$. Low-level exercise performed in conjunction with dipyridamole has been demonstrated to decrease side 
effects and radiotracer uptake in nontarget organs, resulting in higher-quality images. Patients who are ambulatory and do not have a LBBB or paced ventricular rhythm may walk on the treadmill at $2.74 \mathrm{~km} / \mathrm{h}(1.7 \mathrm{mph})$ with $0 \%$ elevation for 4-6 min at the completion of the dipyridamole infusion. During this time, the radiotracer should be injected (35 min after the dipyridamole infusion) and the patient should walk an additional 2 min after injection to ensure adequate tracer uptake in the myocardium. Other suggested protocols for performing exercise in conjunction with dipyridamole include completion of a standard Bruce protocol after infusion. As with any other new or different procedure, thorough research should be performed before such protocols are used within the lab to ensure efficacy and safety to patients $(3,6-8,10)$.

Common side effects of dipyridamole include flushing, chest pain, headache, dizziness, hypotension $(\sim 50 \%$ of patients), and atrioventricular block ( $\sim 2 \%$ of patients). Side effects after dipyridamole may persist 15-25 min after infusion and may vary significantly from patient to patient. Patients may also experience a slight increase in heart rate and a modest decrease in both systolic and diastolic blood pressures. In patients with persistent side effects or clinical symptoms, aminophylline (125-250 mg) may be administered intravenously $2 \mathrm{~min}$ after injection of the radiotracer. Aminophylline should also be injected if postischemic electrocardiogram changes occur. If patients experience chest pain during or after infusion of dipyridamole and administration of aminophylline does not alleviate it, sublingual nitroglycerin, at $0.4 \mathrm{mg}$ every $5 \mathrm{~min}$ for a total of 3 doses, may also be given $(3,6-8,10)$. Dipyridamole should be stopped early if any of the following occur: severe hypotension, severe chest pain with ST depression of $2 \mathrm{~mm}$ or greater, complete heart block, symptomatic second-degree heart block, signs of poor perfusion, patient request, or wheezing $(3,6-8,10)$.

\section{Regadenoson}

Regadenoson is an $\mathrm{A}_{2 \mathrm{~A}}$ agonist that causes vasodilation by activating the $\mathrm{A}_{2 \mathrm{~A}}$ receptors and increasing coronary blood flow in the same manner as adenosine and dipyridamole. Within 1-4 min after administration of regadenoson, the maximal plasma concentration is reached. Regadenoson

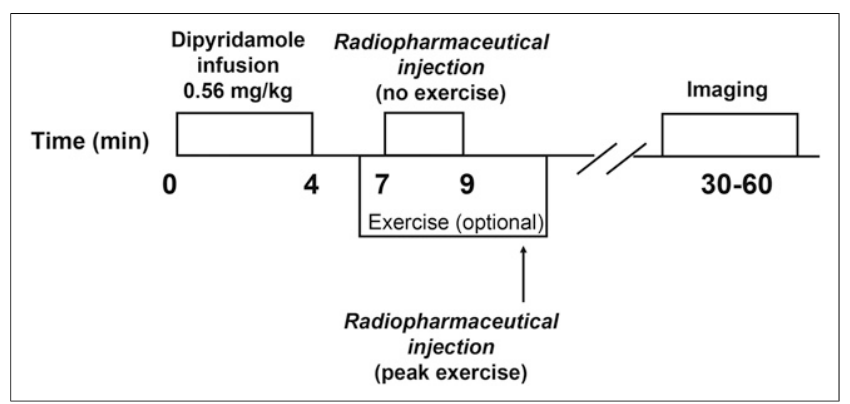

FIGURE 2. Dipyridamole infusion protocol. has 3 half-lives. The initial phase is approximately $2-4 \mathrm{~min}$ and includes onset of the pharmacodynamic response. The intermediate phase, with a half-life of approximately $30 \mathrm{~min}$, coincides with loss of the pharmacodynamic effect. The terminal phase has a half-life of around $2 \mathrm{~h}$ and is characterized by a decline in plasma concentration $(3,6-8,11)$.

Regadenoson is used in patients with baseline electrocardiogram abnormalities such as a LBBB, ventricular pacing, and Wolff-Parkinson-White syndrome. It is also used in patients less than $1 \mathrm{~d}$ following an acute myocardial infarction who are clinically stable, patients who present in the emergency department following acute coronary syndrome, patients who cannot exercise because of pulmonary, peripheral vascular, musculoskeletal, or mental conditions, and patients who are unwilling to exercise. As with exercise stress testing, $\beta$-blockers, nitrates, and calcium antagonists have been reported to decrease the accuracy of diagnosis with the use of vasodilators in stress tests $(3,6-8,11)$.

Contraindications to regadenoson stress testing include second- or third-degree atrioventricular block in the absence of a pacemaker or sick sinus syndrome, consumption of caffeine within $12 \mathrm{~h}$ of administration, asthma with wheezing, use of aminophylline within $24 \mathrm{~h}$, a systolic blood pressure of less than $90 \mathrm{~mm} \mathrm{Hg}$, recent use of dipyridamole or dipyridamole-containing medications (i.e., Aggrenox), known hypersensitivity to adenosine, unstable acute myocardial infarction or acute coronary artery syndrome, known hypersensitivity to regadenoson, and sinus bradycardia with a heart rate of less than 40 beats per minute.

Regadenoson is supplied as a single-use prefilled syringe with a standard dose of $0.4 \mathrm{mg} / 5 \mathrm{~mL}(0.08 \mathrm{mg} / \mathrm{mL})$. It should be administered intravenously over $10 \mathrm{~s}$, immediately followed by a 5 -mL flush of normal saline. Twenty seconds after regadenoson administration, the radiotracer should be injected (Fig. 3) (3,6-8,11).

Exercise stress may be performed in conjunction with regadenoson in those patients unable to reach adequate (symptom-limited) stress or 5-6 metabolic equivalents of exertion. However, exercise should be avoided in patients with a LBBB or a paced ventricular rhythm. If exercise is performed in conjunction with regadenoson, the protocol should be based on the patient's physical capabilities. Regadenoson should be injected following the standard infusion protocol, and exercise should be terminated when the

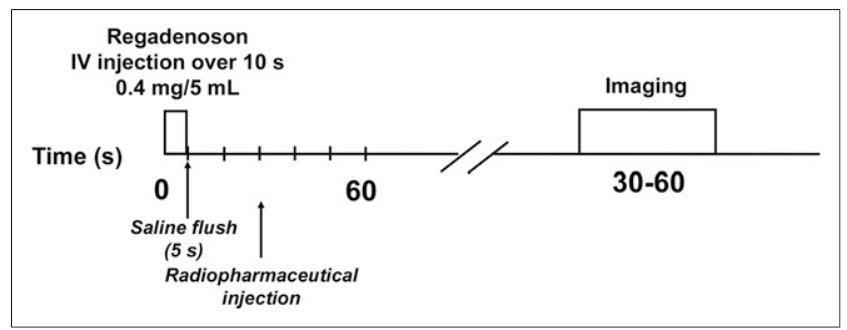

FIGURE 3. Regadenoson infusion protocol. IV = intravenous. 
patient has become symptom-limited or unable to continue $(3,6-8,11)$.

After regadenoson injection, if a patient experiences severe hypotension, severe chest pain with ST depression of $2 \mathrm{~mm}$ or greater, complete heart block, new-onset atrial flutter or atrial fibrillation, symptomatic second-degree heart block, signs of poor perfusion, or wheezing, administration of aminophylline (50-250 mg via intravenous injection of 50-100 mg over 30-60 s) should be considered. If the patient has a seizure, aminophylline should not be administered because of the increased seizure risk associated with regadenoson and its known risk of lowering the seizure threshold in certain patients $(3,6-8,11)$. Common side effects of regadenoson include dyspnea, headache, flushing, chest discomfort, angina pectoris (chest pain), and ST-segment depression.

Adverse reactions associated with regadenoson are usually mild and resolve within 15-30 min after infusion. Headaches have been demonstrated to last the longest of all known side effects without treatment. If side effects persist, administration of aminophylline $(50-250 \mathrm{mg}$ via intravenous injection of $50-100 \mathrm{mg}$ over $30-60 \mathrm{~s}$ ) should be considered. Approximately 3\% of patients experience first-degree atrioventricular block, with $0.1 \%$ experiencing second-degree atrioventricular block. In clinical trials of regadenoson, these rhythm changes did not need to be reversed. Hemorrhagic and ischemic cerebrovascular accidents have also been demonstrated to occur in some patients receiving regadenoson $(3,6-8,11)$.

\section{Dobutamine}

Dobutamine is used primarily in patients unable to exercise and who have contraindications to vasodilators used in conjunction with MPI. Dobutamine, unlike adenosine, dipyridamole, and regadenoson, is not a vasodilator. Dobutamine is a positive inotrope, resulting in direct $\beta_{1}$ and $\beta_{2}$ receptor stimulation, and has a half-life of approximately $2 \mathrm{~min}$. Infusion of this agent results in a dose-related increase in heart rate, blood pressure, and myocardial contractility, which, based on the principles of coronary flow reserve, increase regional myocardial blood flow. Similarly, subepicardial and subendocardial blood flow in vascular beds supplied by normal coronary arteries is increased and is dose-related. The increase in blood flow to vascular beds supplied by stenosed arteries is minimal, with most of the increase occurring within the subepicardium. This discrepancy in blood flow allows for the evaluation of myocardial ischemia when used in conjunction with MPI $(3,6-8,12)$.

The contraindications for dobutamine infusion include myocardial infarction less than $1 \mathrm{wk}$ prior to the test; unstable angina; a prior history of tachycardia, atrial tachyarrhythmia, or uncontrolled ventricular response; severe aortic stenosis; significant left ventricular outflow tract obstruction; severe hypertension; and significant aneurysm or dissection. Patients should not receive dobutamine if they are being treated with $\beta$-blockers (within 48-72 h) or have a LBBB, permanent

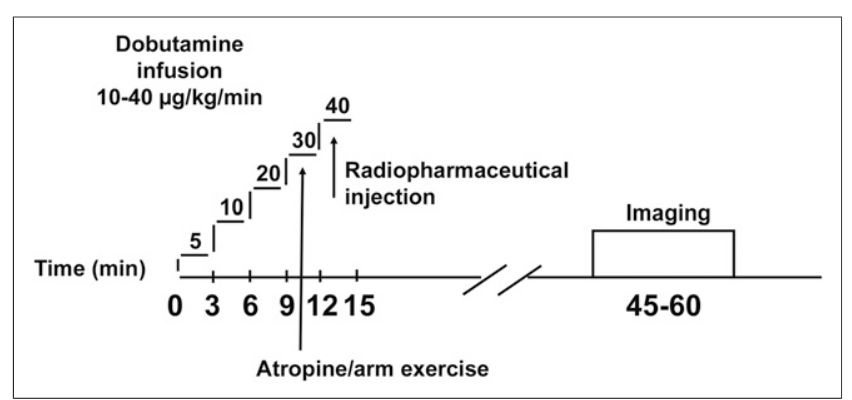

FIGURE 4. Dobutamine infusion protocol.

pacemaker, or Wolfe-Parkinson-White syndrome because a false-positive result may occur.

Dobutamine should be infused intravenously at a dose rate of 5-10 $\mu \mathrm{g} / \mathrm{kg} / \mathrm{min}$ and increased every $3 \mathrm{~min}$ to 20,30 , and $40 \mu \mathrm{g} / \mathrm{kg} / \mathrm{min}$, respectively. The dose increase should be determined by the patient's heart rate and physical response to the medication. The target endpoint for dobutamine is for the patient to achieve at least $85 \%$ of the patient's MPHR. Once this target is achieved, the radiotracer should be injected and dobutamine infusion terminated (Fig. 4) (3,6-8, 12). If the patient does not reach the target heart rate, administration of atropine or use of arm or leg exercises should be considered in order to increase the heart rate. After an attempt at either or both of these interventions, termination of the protocol should be considered if the target heart rate is not achieved in order to avoid a false-negative result $(3,6-8,12)$.

Early termination of the dobutamine infusion should occur for ventricular tachycardia or ST-segment depression, which is more likely to occur with dobutamine than with the other pharmacologic stress agents. Other indications for early termination include moderate to severe angina, a decrease in systolic blood pressure of greater than $20 \mathrm{~mm}$ $\mathrm{Hg}$ from baseline when accompanied by other evidence of ischemia, patient request, signs of poor perfusion, a hypertensive response, increasing chest pain, ST or QRS changes, development of a bundle branch block or intraventricular conduction delay that cannot be differentiated from ventricular tachycardia, ST elevation in leads without diagnostic $\mathrm{Q}$ waves, and marked dyspnea $(3,6-8,12)$.

Approximately $75 \%$ of patients have been demonstrated to experience an adverse reaction during infusion of dobutamine. The most common side effects are palpitations, chest pain, headache, flushing, dyspnea, and significant supraventricular or ventricular arrhythmias. Also, one third of patients have been demonstrated to experience ischemic ST-segment depressions. Side effects may be treated with a short-acting $\beta$-blocker administered intravenously over $1 \mathrm{~min}(3,6-8,12)$.

\section{CONCLUSION}

Stress testing has been used for over $60 \mathrm{y}$ in the evaluation of symptomatic patients (3). Both exercise and 
pharmacologic stress (vasodilator and ionotropic) are performed routinely in conjunction with SPECT and PET MPI. Suboptimal or inadequate stress testing may lead to a falsenegative MPI and poorly impact clinical decision making and patient outcomes $(3,6-8)$. Therefore, it is imperative technologists are well educated regarding considerations for stress testing used in conjunction with MPI, including the principles, indications, contraindications, patient preparation, and protocols for exercise stress testing; the contraindications, administration protocols, and side effects for performing vasodilator (adenosine, dipyridamole, and regadenoson) stress testing; and the contraindications, administration protocols, and side effects for performing dobutamine stress testing.

\section{DISCLOSURE}

No potential conflict of interest relevant to this article was reported.

\section{REFERENCES}

1. Wasserman K, Hansen JE, Sue DY, et al. Principles of Exercise Testing and Interpretation. 5th ed. Philadelphia, PA: Wolters Kluwer/Lippincott Williams \& Wilkins; 2012.
2. Gibbons RJ, Balady GJ, Bricker JT, et al. ACC/AHA 2002 guideline update for exercise testing: summary article, a report of the American College of Cardiology/American Heart Association Task Force on practice guidelines (Committee to update the 1997 exercise testing guidelines). Circulation. 2002;106:18831892 .

3. Henzlova MJ, Duvall WL, Einstein AJ, Travin MI, Verberne HJ. ASNC imaging guidelines for SPECT nuclear cardiology procedures: stress, protocols, and tracers. J Nucl Cardiol. 2016;23:606-639.

4. Myers J, Arena R, Franklin B, et al. Recommendations for clinical exercise laboratories: a scientific statement from the American Heart Association. Circulation. 2009;119:3144-3161.

5. 2. Fletcher GF, Ades PA, Kligfield P, Arena R, Balady GJ, Bittner VA, et al. Exercise standards for testing and training: a scientific statement from the American Heart Association. Circulation. 2013;128:873-934.

6. Farell MB. Myocardial Perfusion Imaging 2015: Quality, Safety, and Dose Optimization. Reston, VA: Society of Nuclear Medicine and Molecular Imaging Technologist Section; 2016.

7. Farrell MB, Mantel ES, Basso DA, Thomas KS, Kerr BR. Quick Reference Protocol Manual for Nuclear Medicine Technologists. Reston, VA: Society of Nuclear Medicine; 2014

8. Heller GV, Hendel R, Mann A. Nuclear Cardiology: Technical Applications. New York, NY: McGraw Hill; 2009.

9. Adenoscan ${ }^{\circledR}$ (adenosine) injection [package insert]. Northbrook, IL: Astellas Pharma US, Inc.; 2014.

10. Persantine ${ }^{\circledR}$ (intravenous) injection [package insert]. Burlington, Ontario, Canada: Boehringer Ingelheim Canada Ltd.; 2012.

11. Lexiscan ${ }^{\circledR}$ (regadenoson) injection [package insert]. Northbrook, IL: Astellas Pharma US, Inc.; 2018.

12. Dobutamine injection [package insert]. Bedford, OH: Bedford Laboratories; 2013. 